\title{
Equilibrium Ionization and Non-Equilibrium Ionization Plasma Models
}

\author{
Hans Böhringer \\ Max-Planck-Institut für extraterrestrische Physik, Giessenbachstrasse, D-85740 \\ Garching, Germany
}

\begin{abstract}
This paper provides a brief review on ionization and radiation models of an optically thin, hot plasma and their application to the interpretation of observations of the Local Bubble. The basic structure and atomic physics ingredients of the models are outlined. Some general characteristic results of ionization equilibrium and non-equilibrium calculations are shown. Recent progress in plasma model codes is reviewed. The interpretation of the observations of absorption lines and emission spectra from the Local Bubble is discussed in the framework of ionization equilibrium and non-equilibrium ionization models with particular reference to the fast adiabatic cooling model of Breitschwerdt and Schmutzler.
\end{abstract}

\section{Introduction}

The lack of observed absorption towards nearby stars (see York \& Frisch 1983 and P. Frisch in this volume) and the structure of the neutral hydrogen distribution as observed in $21 \mathrm{~cm}$ (see C. Heiles this volume) gives a first impression that the solar system is located in a partly evacuated cavity that might result from an expanding hot superbubble in which we are located. Convincing evidence for a Local Hot Bubble and a physical understanding of this environment come from the observation of soft X-ray emission and UV absorption lines. It is the interpretation of these EUV and X-ray data that leads to a knowledge of physical parameters like temperature, plasma density, and pressure in our local galactic environment. A physical model of the Local Bubble can therefore be tested in the first place by its ability to reproduce the spectral features observed in the UV and soft X-rays.

In this paper we review the principles of plasma emission models and their application to the Local Bubble observations. It turns out that the observed features can very roughly be reproduced by two different model sets: ionization equilibrium models with a fairly hot temperature of the order of $10^{6} \mathrm{~K}$ and fast cooling non-equilibrium ionization models with temperatures as low as a few $10^{4} \mathrm{~K}$. An illustration of the differences between the two model families is the main goal of this paper. 


\section{The Basic Structure of Plasma Emission Models}

The atomic physical processes involved in the plasma models comprise the processes controlling the ionization of the constituents of the interstellar plasma and events leading to the excitation and radiative deexcitation of atomic levels of the ion species. For the hot interstellar medium (ISM) the case is greatly simplified in two ways by the fact that the plasma is very tenuous. Firstly, the plasma is "optically thin" such that a photon once emitted has a negligible chance to be reabsorbed (except for hydrogen Ly- $\alpha$ at low temperatures). This further implies that the radiation spectrum has no feedback to the ionization structure. Secondly, the collision frequency is so low that excited states are deexcited by radiation rather than by collisions even for "strongly forbidden" transitions. The line emission rate is thus given by the rate of collisional excitation. The latter simplification is called "coronal approximation" and certainly holds for electron densities $\leq 10^{4} \mathrm{~cm}^{-3}$.

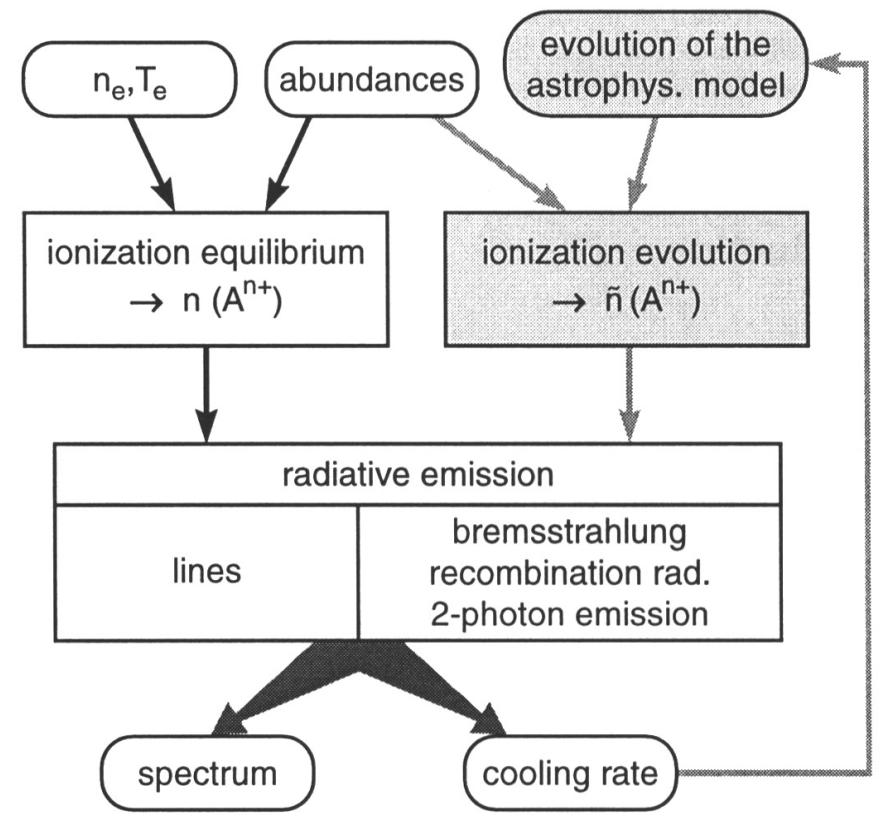

Fig. 1. Schematics of the basic structure of plasma emission models: the white boxes and black arrows outline the structure of equilibrium models; the grey boxes indicate the modifications necessary for time dependent non-equilibrium calculations.

The basic structure of plasma emission models is sketched in Fig. 1. For ionization equilibrium models the only input parameters are the plasma tem- 
perature and the abundances of the elements. The first part of the model consists of the calculation of the ionization structure. In equilibrium, for each consecutive ion pair the ionization rate has to equal the recombination rate as given by equ. (1). This implies the solution of the set of linear equations to determine the relative abundance of each ion of an element:

$$
\begin{gathered}
{\left[I^{n+}\right] n_{e}\left(C_{c i}+C_{a i}\right)+\left[I^{n+}\right]\left(\left[H^{+}\right] X_{c e 1}+\left[H e^{+}, H e^{++}\right] X_{c e 2}\right)=} \\
{\left[I^{(n+1)+}\right] n_{e}\left(C_{r r}+C_{d r}\right)+\left[I^{(n+1)+}\right]\left([H] X_{c e 3}+[H e] X_{c e 4}\right)}
\end{gathered}
$$

Several processes contribute to the ionization and recombination rate. The ionization comprises direct electron impact ionization $\left(C_{c i}\right)$, electron impact excitation to an autoionizing state with subsequent autoionization $\left(C_{a i}\right)$, and ionizing charge exchange with $\mathrm{H}^{+}, \mathrm{He}^{+}$, and $\mathrm{He}^{++}$(with the rates $X_{c e 1}$ and $X_{c e 2}$, respectively). Direct ionization is the dominant process. Recombination includes radiative recombination $\left(C_{r r}\right)$ and dielectronic recombination $\left(C_{d r}\right)$. In the second processes the recombining collision complex is energetically stabilized by the excitation of a second bound electron which will subsequently radiate the excess energy. This is a resonant process which naturally has a strong energy dependence. It is most important for the more complex ions with a large number of electrons (e.g. $F e$ ). It can dominate the recombination process in certain energy (temperature) ranges. Also charge exchange with $H$ and $\mathrm{He}$ (rates $X_{c e 3}$ and $X_{c e 4}$ ) contribute to the effective recombination.

Once the density of each relevant ion species is determined by the elemental abundances and the set of equations (1), the spectral contribution of each ionic species can be calculated. There are four processes to be considered, line emission from collisionally excited states and three continuum emission processes: free-free radiation, recombinative radiation, and two-photon emission. The latter process originates from collisional excitation of energy levels with forbidden transitions which can only radiatively decay by the simultaneous emission of two photons (in particular transitions from $n S$ to $(n-1) S$ orbitals).

All these processes governing the ionization structure and spectral emission involve the collision of electrons with ions with rate equations of the basic form:

$$
R=n_{e} n_{I} C_{I x}=n_{e}^{2}\left[\frac{n_{I}}{n_{E}}\right]\left[\frac{n_{E}}{n_{e}}\right] C_{I x}(T),
$$

where $n_{E}$ and $n_{I}$ are the densities of the element and ion, respectively, and $C_{I x}$ are the rate coefficients of the relevant processes. Thus all rates are proportional to the plasma density parameter, $n_{e}^{2}$, and otherwise depend only on the relative abundances and the temperature dependent rate coefficients. Therefore the ionization structure and the form of the emission spectrum can be evaluated independently from the plasma density. The normalization of the emitted spectrum is then obtained from the emission measure, $E$, calculated for the astrophysical model as 


$$
E=\int n_{e}^{2} d l
$$

The strategy for the calculation of non-equilibrium ionization models is also sketched in Fig. 1. Briefly, the ionization structure is determined by the ionization history: ionization and recombination rates for all relevant ion pairs and the resulting change in the ionization structure have to be calculated along with the astrophysical model in a time dependent way. The radiation can still be calculated independently once the ionization structure is determined. But if radiative cooling is important in the astrophysical model, the total cooling power of the emitted spectrum has also to be calculated simultaneously with the evolving dynamical model.

The calculation of the ionization-radiation model is a very intense bookkeeping task of the rates of all the important atomic processes. Several widely used plasma emission codes have been developed over the last two or three decades which by today keep track of thousands of atomic collision rates. Pioneering work was in particular done by Cox, Tucker, Raymond and Smith. There are currently four openly distributed, frequently used plasma codes which experience a continuous development:

- RS, RSB code: by Raymond \& Smith (1977), Raymond 1988, Brickhouse, Raymond \& Smith (1995a), Raymond \& Brickhouse (1996)

- Mewe, MEKA, MEKAL, SPEX codes: by Mewe et al. (1981, 1985), Gronenschild \& Mewe (1978), Mewe (1990, 1991), Kaastra \& Mewe (1994), Mewe, Kaastra, \& Liedahl (1995)

- Masai code: by Masai (1984), Masai \& Kato (1987), Masai (1994a,b)

- LF code: by Landini and Monsignori Fossi (1970, 1990, 1991), Monsignori Fossi \& Landini (1994).

A recent discussion on a comparison of these codes is given in the Legacy Journal (Brickhouse et al. 1995b). More codes have been constructed, that were used mostly by the authors themselves as for example Cox \& Tucker (1969), Tucker \& Koren (1971), Cox \& Daltabuit (1971), Cox (1972), Shapiro

\& Moore (1976, 1977), Kato (1976), Gaetz \& Salpeter (1983), Böhringer \& Hensler (1989), Schmutzler \& Tscharnuter (1993), and Sutherland \& Dopita (1993).

\section{Ionization Structure of Equilibrium Models}

Some generally interesting results concern for example the calculated ionization structure for oxygen as a function of temperature. Results taken from Böhringer \& Hensler (1989) are shown in Fig. 2. The lithium-like oxygen ion $O V I$, which is marked in the plot, has a single outer electron in the L-shell and is characterized by a very large photon absorption cross section (in the UV). It also has a very peaked abundance profile as a function of temperature. 
Both properties are very important: the discovery of this ion in absorption in the ISM with the Copernicus satellite (Jenkins \& Meloy 1974) has led to the implication of the existence of a hot ISM phase with coronal temperatures. The narrow range over which this ion appears abundant (see Fig. 2) implies a plasma temperature of about $2-5 \cdot 10^{5} \mathrm{~K}$ for the local ISM.

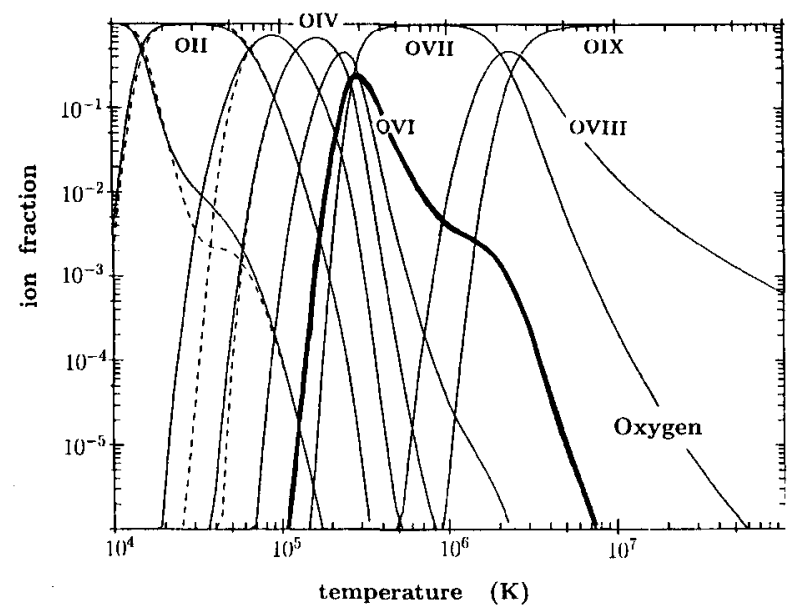

Fig. 2. Equilibrium ionization structure of oxygen as a function of temperature calculated from the data of Arnaud \& Rothenflug (1985). The thick line marks the abundance of $O^{5+},(O V I)$. The dashed lines show the calculations without charge exchange reactions.

Similarly the ions $N V$ and $C I V$ - also lithium-like - have been observed in the line of sight through the galactic halo (e.g. Savage \& DeBoer 1981, Savage \& Massa 1987). They indicate temperatures in the interval $1.5-2.5 \cdot 10^{5} \mathrm{~K}$ and $0.8-1.3 \cdot 10^{5} \mathrm{~K}$, respectively.

\section{Recent Progress in Plasma Codes}

How reliable are the results for theoretically calculated spectra? One may get an idea of the accuracy of the models by inspecting the effects that two recent major revisions had on the results from plasma codes. The remaining uncertainties in the present models may be of similar order in the worst case.

The first improvement concerns the calculation of the ionization structure of the heavy elements, in particular iron. Arnaud \& Raymond (1992) have compiled new experimental data from crossed beam techniques, photoionization cross sections, and theoretical calculations for dielectronic recombination and recalculated a scheme for the ionization and recombination structure of iron. The results are major changes in the ionization structure of iron as a function of temperature. The differences are mostly due to the uncertainties in the very complex dielectronic recombination process of iron. With these changes some of the puzzles in the ion abundances deduced from solar X- 
ray spectra can be explained, but also some puzzles remain (see Arnaud \& Raymond 1992).

Masai (1997) has studied the effects of the use of different ionizationrecombination data sets from Arnaud \& Rothenflug (1985), Masai (1994), Kato, Masai, Arnaud (1991), Arnaud \& Raymond (1992) for spectra of a plasma with a temperature around $1 \mathrm{keV}$ and about solar abundances. He finds that the differences between the results from the different codes are: $\Delta\left(T_{e}\right) \sim 15 \%$ for the temperature, $\Delta(Z) \geq 30 \%$ for the abundances, and there are up to factors of 2 variations in the emissivity of the plasma in the spectrum around $1 \mathrm{keV}$. Masai (1997) argues that not all the uncertainties in the ionization structure of iron may have been resolved in the latter revisions and therefore one may conclude that the remaining uncertainties could be of almost similar order as the differences outlined.

The second revision concerns the L-shell line excitation rates of the element iron. The reconsideration of the excitation rates was triggered by the study of early spectra of galaxy clusters taken by the ASCA satellite. Fabian et al. (1994) found that the $F e$ (3-2) and $F e$ (4-2) transition line blends could not be fitted simultaneously by single- or multi-temperature models in the high quality spectrum taken of the Centaurus galaxy cluster. Reconsideration of the transition probabilities of the iron L-shell lines by Liedahl et al. (1995) showed that the major problems lay in the inaccuracy of the previously calculated atomic data. Recalculation of the line strength could solve the major discrepancies and led to the development of the MEKAL code (Mewe, Kaastra, \& Liedahl 1995). The results are now included in most modern codes. The effect of the improvements are changes in the intensities of iron line blends around $1 \mathrm{keV}$ of up to a factor of two.

These improvements have been driven by the wish to understand the observed astrophysical spectra and have essentially focussed in the element iron. The progress goes on line with the technical improvement of the observations. For the earlier X-ray spectroscopy at higher energies with the EXOSAT or GINGA observatories, the $\mathrm{K}$-shell lines of iron were most important and easy to model. With ASCA the other heavy element K-shell lines are well observed and the iron L-shell lines are prominent in the observational window. Hence the need to understand the more complicated iron L-shell physics. With further progress in the detectors down to lower energies the L-shell lines of elements like $S, S i, M g, N e, O$ and eventually the M-shell lines of $F e$ are becoming important. DXS observations of such lines were reported by Sanders at this conference and new calculations of the line strengths of the relevant ions are in progress by Liedahl et al.. There is great hope to gain a better understanding of the physical state of the Local Bubble from the detailed analysis of such EUV and very soft X-ray spectra. 


\section{Non-Equilibrium Ionization Models}

There are several types of equilibria that have to be established for ionization equilibrium models to apply: (i) electrons and ions have to be thermalized to unique electron and ion temperatures, $T_{e}$ and $T_{i}$; (ii) $T_{e}$ and $T_{i}$ have to come to equilibrium (which may not be the case e.g. in young supernova remnants); (iii) the ionization and recombination rates have to come to a balance. This requires that the atomic collision processes are faster than any other change of the considered environment. The first two processes are relatively fast and controlled by Coulomb collisions or even faster plasma effects. It is the third type of deviation from equilibrium that is of concern here in the context of the Local Bubble.

In particular the cooling of the ISM plasma can be faster that the recombination of some ions with important cooling lines. In this case the ionization structure is not equal to the ion abundances calculated for ionization equilibrium. As an example we can show this effect for the recombination of $C V: C V+e \rightarrow C I V+h \nu$ for which $t_{\text {recomb }}\left(3 \cdot 10^{5} \mathrm{~K}\right)=$ $\alpha_{\text {recomb }}^{-1} \cdot n_{e}^{-1} \sim 20000 \mathrm{yrs}\left(\frac{1 \mathrm{~cm}^{-3}}{n_{e}}\right)$ which is longer than the plasma cooling time $t_{\text {cool }}=\frac{1.5 n k T}{n_{e} n_{p} \Lambda(T)} \sim 6600 \mathrm{yrs}\left(\frac{1 \mathrm{~cm}^{-3}}{n_{e}}\right)$. In a cooling plasma the recombination of $C V$ will lag behind and the plasma will contain less of the strongly emitting lithium like $C I V$. The paper by Sutherland \& Dopita (1993) shows a very nice diagnostic plot in Fig. 15 which gives a comparison of the recombination times versus the cooling time for a large set of relevant ions.

In early calculations by Shapiro and Moore (1976), for example, the authors have shown that in the cooling of hot plasma (starting with equilibrium conditions well above $10^{6} \mathrm{~K}$ ) non-equilibrium effects set in at temperatures below about $3-5 \cdot 10^{5} \mathrm{~K}$. These effects are strong enough to change the cooling rate of the plasma as shown in Fig. 3. The non-equilibrium model contains more highly ionized ion species with less electrons in the outer shells which can provide the strongest emission lines. Therefore the non-equilibrium ionization model provides a less fast cooling than calculated for the equilibrium case. The retarded recombination also leaves its trace in the plasma spectrum. There is a substantial energy release connected with the delayed recombining ions which shows up in a saw-teeth continuum structure in the upper energy part of the spectrum.

A more recent and more detailed calculation of the ionization structure of cooling plasma is given by Schmutzler and Tscharnuter (1993). One interesting aspect seen in the results of their calculations is the change in the ionization structure of $C, N$, and $O$. Fig. 4 shows for example the ionization structure of oxygen (which should be compared to the equilibrium ionization structure shown in Fig. 2). Above, with only the equilibrium ionization models at hand, we have argued that the abundant observation of the ion $O V I$ in the ISM implies that there is an important, widespread hot ISM phase with 


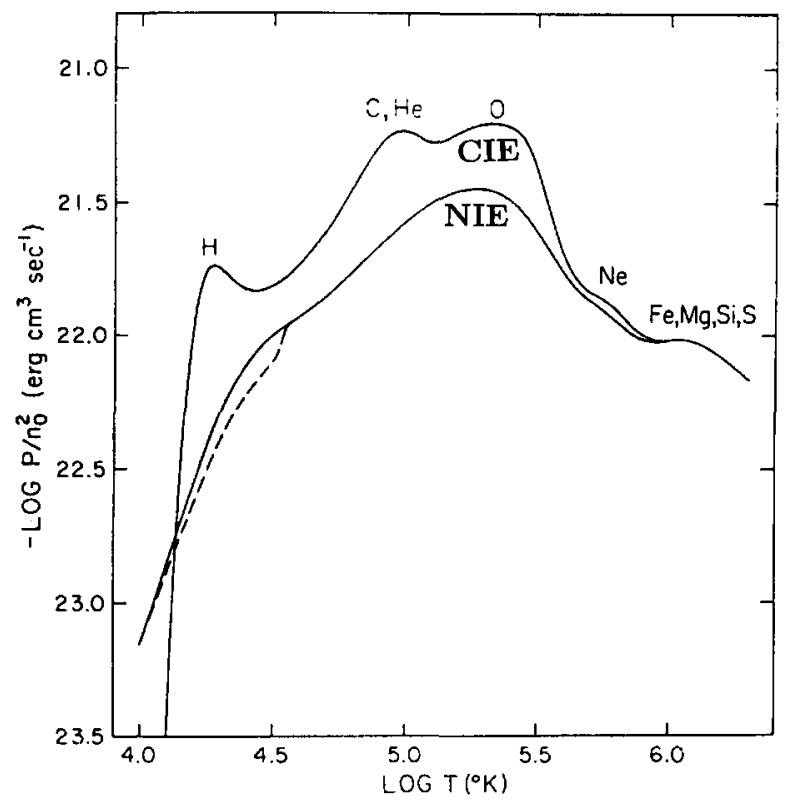

Fig. 3. Cooling coeffcient as a function of $T_{e}$ for the non-equilibrium ionization calculation of cooling plasma from Shapiro \& Moore (1976).

a temperature in the narrow range $2-5 \cdot 10^{5} \mathrm{~K}$. Now in Fig. 4 we note that the "diagnostic ion" $O V I$ is abundant over quite large range of temperatures from about $4 \cdot 10^{5} \mathrm{~K}$ to temperatures as low as $10^{4} \mathrm{~K}$. Also the ion OVII (the He-like species) which is also observed in the local ISM (see contribution by Bowyer et al.) can be found at low temperatures in non-equilibrium models. In a similar way the ions $\mathrm{NV}$ and CIV, observed in the galactic halo, lose their diagnostic power to indicate a very hot galactic halo.

The opposite effect of deviation from ionization equilibrium is observed in models with extra heat input to the plasma, where the heating rate can be faster than the ionization rates. Shapiro and Moore (1977) show such models for example for coronal loops with sudden heating. For the ISM this may be relevant for conductively heated interfaces between the hot ISM and embedded clouds. In a model for such interfaces Böhringer \& Hartquist (1989) showed that non-ionization equilibrium structures can occur at the intermediate temperature ranges (few $10^{5} \mathrm{~K}$ ) which are most important for their effective radiative emission. The principle results of these type of models are characterized by low-ionization stage ions in a hotter environment with an increased contribution of emission lines to the total radiation as compared to continuum processes. Some strong and dominant emission lines can be boosted by up to about two orders of magnitude (e.g. Fig. 6 in Böhringer \& Hartquist 1989). 


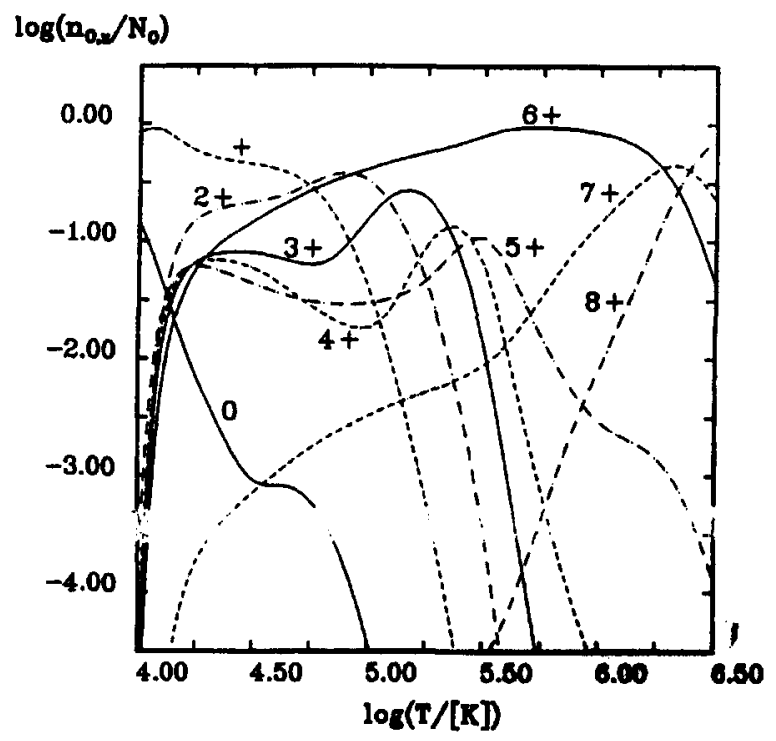

Fig. 4. Non-equilibrium ionization calculation for the ionization structure of Oxygen for cooling plasma from Schmutzler \& Tscharnuter (1993).

\section{Models for the Local Bubble}

To approximately account for the soft X-ray and UV observations of the ISM two families of models are invoked: models near ionization equilibrium at high temperatures (around $10^{6} \mathrm{~K}$ ) and models that start at high initial temperatures (above $10^{6} \mathrm{~K}$ ) but experience a rapid cooling disturbing the ionization balance.

For the first case, early models, as for example Cox \& Anderson (1982), have considered supernova explosions to explain the soft X-ray observation with typical parameters of a gas temperature of $\sim 10^{6} \mathrm{~K}$, a pressure of $P / k \sim 10^{4} \mathrm{~cm}^{-3} \mathrm{~K}$, and an extent of one to several $100 \mathrm{pc}$. More recent models also consider the case of a superbubble with an energy input more than an order of magnitude higher than that of a single supernova (e.g. Innes \& Hartquist 1984, Edgar \& Cox 1993). While in the first model the bubble is fairly young with an age of the order of $10^{5} \mathrm{yr}$, the superbubbles can be inflated by subsequent energy releases and have a much larger age. For the younger bubbles even non-equilibrium ionization effects in the sense of too low ionization stages for the ambient temperature have to be considered (see Cox \& Anderson 1982).

In summary these " $~ 10^{6} \mathrm{~K}$-models" can reproduce the observed Be, B, C band data in soft X-rays. Two problems have been outlined for these models: they produce little emission in the M-band (see Cox \& Anderson 1982, Edgar $\&$ Cox 1993) unless the temperature is raised considerably above $10^{6} \mathrm{~K}$; They imply a large pressure of the LISM which exceeds the pressure deduced for the local cloud by an order of magnitude (e.g. Breitschwerdt 1996). 
As an alternative model Breitschwerdt and Schmutzler (1994) constructed a history of the Local Bubble which consists of the creation of a superbubble in a dense cloudy environment, an out-break with a fast adiabatic expansion, and the escape of some hot ISM in a galactic wind. Compared to the above hot Bubble models, some more degrees of freedom are introduced in particular the ratio of the adiabatic cooling rate versus the radiative cooling rate as a function of time.

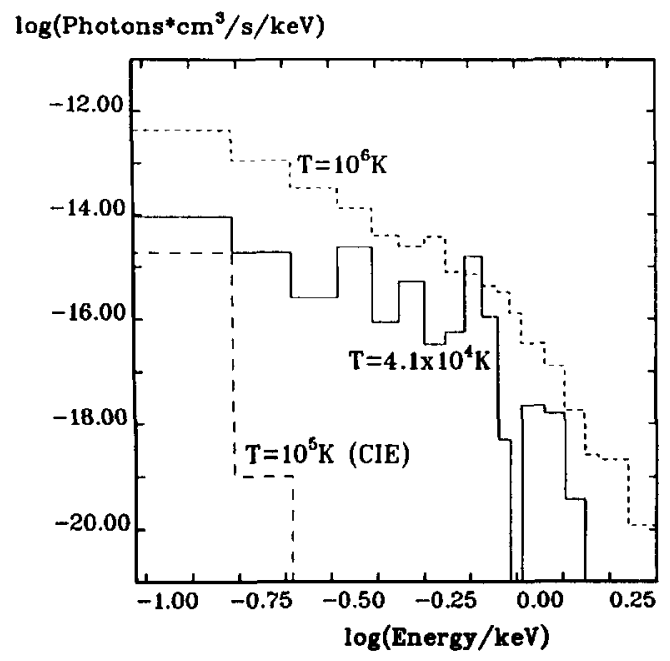

Fig. 5. X-ray spectrum of the non-equilibrium model by Breitschwerdt \& Schmutzler (1994) for the Local Bubble and the galactic halo. The energy window and the resolution corresponds approximately to that of the ROSAT PSPC. The non-equilibrium model for a temperature of $4 \cdot 10^{4} \mathrm{~K}$ is compared to a $10^{6} \mathrm{~K}$ model and an equilibrium model at $10^{5} \mathrm{~K}$. The non-ionization equilibrium model mimics the coronal temperature model in spite of its low temperature.

With appropriately chosen model parameters (initial temperature $=2.5$. $10^{6} \mathrm{~K}$, initial ambient density up to $10^{4} \mathrm{~cm}^{-3}$ ) one finds results that can fit the observations for a present ambient temperature of the Local Bubble as low as a few $10^{4} \mathrm{~K}$. These solutions are characterized by large deviations from ionization equilibrium with most highly ionized species from the initial temperature plasma still present. A large fraction of internal energy of the plasma is stored in the highly ionized species, which can subsequently be released by recombinative radiation. The emission spectra are characterized by an unusually large contribution from recombination radiation, such that the high energy part of the spectrum is completely dominated by recombination photons. Due to this high energy recombination tail the emission spectrum of $4 \cdot 10^{4} \mathrm{~K}$ non-equilibrium plasma can mimic a spectrum of $10^{6} \mathrm{~K}$ plasma in its rough overall shape and hardness of the radiation as shown in Fig. 5. Such a model can roughly reproduce the observed spectra in the soft X-ray and EUV regime as shown in Fig. 6 (taken from Breitschwerdt \& Schmutzler 1994).

As shown in this paper, the introduction of fast cooling non-equilibrium ionization models opens a larger spectrum of scenarios for the explanation 
of the Local Bubble observations. Firm model predictions can, however, be made. One can in the future differentiate between the various models when sufficiently high resolution spectroscopic data become available resolving the lines and recombination radiation features.

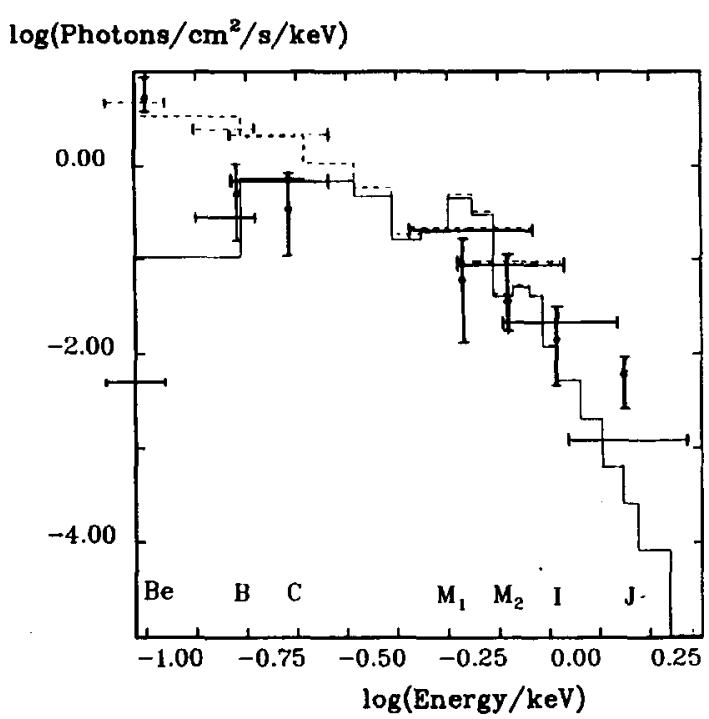

Fig. 6. Comparison of the results for the emission spectrum of the Local Bubble and a galactic wind from the model of Breitschwerdt \& Schmutzler (1994) with the observations in the various EUV and soft X-ray bands. Good agreement can be found for appropriately chosen model parameters (for details see Breitschwerdt \& Schmutzler (1994).

\section{References}

Arnaud, M. \& Rothenflug, R., 1985, A\&AS, 60, 425

Arnaud, M. \& Raymond, J., 1992, ApJ, 398, 394

Böhringer H. \& Hartquist, T.W., 1987, MNRAS, 228, 915

Böhringer H. \& Hensler, G., 1989, A\&A, 215, 147

Breitschwerdt, D., 1996, Space Sci. Rev., 78, 173

Breitschwerdt, D. \& Schmutzler, T., 1994, Nature, 371, 774

Brickhouse, N.S., Raymond, J.C., Smith, B.W., 1995, ApJS,

Brickhouse, N. et al., 1995, LEGACY, Journal of HEASARC at NASA GSFC, 6, 4 Cox, D.P., 1972, ApJ, 178, 143

Cox, D.P. \& Tucker, W.H., 1969, ApJ, 157, 1157

Cox, D.P. \& Daltabuit, E., 1971, ApJ, 167, 113

Cox, D.P. \& Anderson, P.R., 1982, ApJ, 253, 268

Edgar R.J. \& Cox, D.P., 1993, ApJ, 413, 190

Fabian, A.C., Arnaud, K.A., Bautz, M.W., Tawara, Y., 1994, ApJ, 436, L63

Frisch, P.C. \& York, D.G., 1983, ApJ, 271, L59

Gaetz, T.J. \& Salpeter, E.E., 1983, ApJS, 52, 155

Gronenschild, E.H.B.M. \& Mewe, R., 1978, A\&AS, 32, 283

Innes, D.E. \& Hartquist, T.W., 1994, MNRAS, 209, 7 
Kaastra, J.S. \& Mewe, R., 1994, LEGACY , Journal of HEASARC at NASA GSFC, 3,16

Kato, T., ApJS, 30, 397

Kato, T., Masai, K, Arnaud, M., 1991, NIFS-DATA-14, Nat. Inst. Fusion Res., Nagoya

Landini, M. \& Monsignori Fossi, B.C., 1970, A\&AS, 6, 468

Landini, M. \& Monsignori Fossi, B.C., 1990, A\&AS, 82, 229

Landini, M. \& Monsignori Fossi, B.C., 1991, A\&AS, 91, 183

Liedahl, D.A., Osterhold, A.L., Goldstein, W.H., 1995, ApJ, 438, L115

Masai, K., 1984, Astrophys. Space Sci., 98, 367

Masai, K. \& Kato, T., 1987, Phys. Lett. A, 123, 405

Masai, K., 1994a, ApJ, 437, 770

Masai, K., 1994b, JQSRT, 51, 211

Masai, K., 1997, A\&A, preprint

McCammon, D. \& Sanders, W.T., 1990, ARA\&A, 28, 657

Mewe, R., 1990, in Physical Processes in Hot Cosmic Plasmas, W. Brinkmann et al. (eds.), Kluwer, p. 39

Mewe, R., 1991, A\&AR, 3, 127

Mewe, R. \& Gronenschild, E.H.B.M., 1981, A\&AS, 45, 11

Mewe, R., Gronenschild, E.H.B.M., van den Oord, G.H.J., 1985, A\&AS, 62, 197

Mewe, R., Kaastra, J.S., Liedahl, D.A., 1995, LEGACY, Journal of HEASARC at NASA GSFC, 6,16

Monsignori Fossi, B.C. \& Landini, M., 1994, A\&A, 284, 900

Raymond, J.C. \& Smith, B.W., 1977, ApJS, 35, 419

Raymond, J.C., 1988, in Hot Thin Plasmas in Astrophysics, R. Pallavicini (ed.), Kluwer, Dordrecht, p. 3

Raymond, J.C. \& Brickhouse, N.S., 1996, Astrophys. Space Sci., 237, 321

Savage, B.D. \& DeBoer K.S., 1981, ApJ, 243, 460

Schmutzler, T. \& Tscharnuter, W.M., 1993, A\&A, 273, 318

Shapiro, P.R. \& Moore, R.T., 1976, ApJ, 207, 460

Shapiro, P.R. \& Moore, R.T., 1977, ApJ, 217, 621

Shull, J.M., 1981, ApJS, 46, 27

Sutherland, R.S. \& Dopita, M.A., 1993, ApJS, 88, 253

Tucker, W.H. \& Koren, M., 1971, ApJ, 168, 283 